\title{
Endoscopic screening for Barrett's esophagus: while we're in, do we also need to see the stomach and the duodenum?
}

\section{(ㄷ)(1)}

\begin{abstract}
Author
Mihai Ciocîrlan

Institution
Carol Davila University of Medicine and Pharmacy, Fundeni
Gastroenterology and Hepatology Center, Bucharest,
Romania
\end{abstract}

submitted 25.1.2017

accepted after revision 1.2 .2017

\author{
Bibliography \\ DOI https://doi.org/10.1055/s-0043-102937 | \\ Endoscopy International Open 2017; 05: E345-E347 \\ (c) Georg Thieme Verlag KG Stuttgart · New York \\ ISSN 2364-3722
}

\author{
Corresponding author \\ Mihai Ciocîrlan, Carol Davila" University of Medicine and \\ Pharmacy, Fundeni Gastroenterology and Hepatology \\ Center, 258 Fundeni Street, Bucharest, RO 022328 \\ ciocirlanm@yahoo.com
}

In this issue of Endoscopy International Open, the Mayo Clinic team of Dr. Iyer Prasad published a comparative quality assessment of the endoscopy videos recorded in their previous published work on Barrett esophagus screening [1,2]. Their initial work compared the yield of 2 different endoscopic examinations: unsedated transnasal esophagoscopy (UTNE) and sedated esophago-gastro-duodenoscopy (sEGD).

In UTNE, the tube is covered with a single-use plastic sheath that makes sterilization between procedures unnecessary (Endosheath, TNE-5000, Vision Sciences, Orangeburg, NY, USA). The endoscope's sheath has an outer parallel biopsy channel made from the same material. Due to its short length, it can only examine the esophagus. In SEGD, a standard highdefinition 9.8-m endoscope (GIF-180, Olympus America, Center Valley, PA, USA) is used.

Transnasal esophagoscopy (TNE) was used both in hospital (huTNE) and in a community setting through a specially designed mobile research vehicle (muTNE). Esophago-gastroduodenoscopy (EGD) was used in hospital. The screening population consisted of individuals aged older than 50 , with or without gastroesophageal reflux disease (GERD) symptoms.

The conclusion of the first study [1] was that both hospital and mobile van uTNE screening had comparable clinical effectiveness to SEGD (complete examination of the esophagus in $96 \%$ to $100 \%$ of cases, and similar participation rates [ $40 \%$ to $45 \%$ ] and safety profile). Mean recovery time with uTNE was significantly shorter compared to SEGD (15 minutes versus 1 hour). Rates of successful biopsy acquisition were significantly lower in the UTNE groups compared to SEGD due to inability to advance the TNE scope with the biopsy sheath through narrow nasal passages and patient intolerance ( $80 \%$ versus $100 \%$ ), but switching to a smaller sheath allowed subsequent esophageal examination [1,3]. Esophagitis was diagnosed in $32 \%$ and Barrett esophagus in $8 \%$ of screened individuals [1].

The current study [2] published in Endoscopy International Open expands upon the previous findings and concludes that the overall quality and gastroesophageal junction visualization of UTNE and SEGD are comparable.

To better understand the big picture in which this paper appears, we need to ask ourselves a few questions.

\section{Do we need to screen for Barrett's esophagus?}

There are currently 3 published guidelines, by the American Society of Gastrointestinal Endoscopy (ASGE) in 2012, the British Society of Gastrointestinal Endoscopy (BSG) in 2014 and the American College of Gastroenterology (ACG) published in $2016[4,5,6]$.

All guidelines note that the current risk factors for Barrett's esophagus are age older than 50 , white race, male sex, smoking, obesity, chronic GERD symptoms and family history of first degree of Barrett's esophagus or esophageal adenocarcinoma.

The guidelines state that endoscopic screening of the general population is not justified when there are no risk factors or only 1 risk factor is noted. Screening can be considered when multiple concomitant risk factors are present, especially in men with chronic GED symptoms.

However, ASGE guideline states that "endoscopic screening for $B E$ is controversial because no randomized, controlled trials (RCTs) have demonstrated a decrease in mortality, either in general or from EAC, as a result of screening". 


\section{If screening for Barrett's esophagus is decided, do we have to use endoscopy or are there other means?}

Capsule endoscopy [7, 8], string capsule endoscopy [9], and Cytosponge [10] are alternative means for Barrett's esophagus screening, with reported sensitivities $60-70 \%$ and specificities $85-100 \%$, when endoscopy is viewed as a confirmatory test. Capsule endoscopy may also explore other segments of the $\mathrm{Gl}$ tract besides the esophagus.

\section{If endoscopy is to be used for Barrett's esophagus screening, is small-caliber unsedated transnasal endoscopy (UTNE) comparable to standard sedated endoscopy (sEGD)?}

Besides the abovementioned study by Prasad et al. [1], there were 2 other comparative randomized crossover studies comparing uTNE with sEGD. Their population consisted of patients screened for Barrett's esophagus or with Barrett's esophagus surveillance.

In the first paper by Jobe et al. [11], uTNE was performed with a 5.1-mm diameter, 65-cm long endoscope (Olympus, USA), which was able to visualize the esophagus and the stomach, but not the duodenum. UTNE and sEGD detected Barrett's esophagus and dysplasia with similar sensitivity, with moderate agreement between the two methods $(k=0.59)$. $71 \%$ of patients preferred uTNE.

In the second paper by Schariff et al. [12], uTNE was performed with a 5.9-mm diameter, standard-length endoscope (EG530N, Fujinon, USA), which can be used to perform a full EGD. UTNE had a sensitivity and specificity for Barrett's esophagus of $98 \%$ and $100 \%$, respectively, while the sensitivity and specificity for detecting intestinal metaplasia were $96 \%$ and $100 \%$, respectively. Fifty-nine percentof patients preferred UTNE.

In conclusion, uTNE compared favorably to SEGD for Barrett's esophagus screening and surveillance. ACG guidelines state that "UTNE can be considered as an alternative to conventional upper endoscopy for Barrett's esophagus screening”.

Current manufacturers offer small-caliber pediatric gastroscopes with $4.9 \mathrm{~mm}$ to $6.00 \mathrm{~mm}$ diameter range, which have been used extensively for uTNE examination in different settings $[13,14]$.

\section{If unsedated transnasal endoscopy is to be used for Barrett's esophagus screening, would it be desirable to see the stomach and duodenum also?}

In a recent screening study in a population of asymptomatic individuals (mean age 52, range 26-83) where EGD was used as a confirmatory test, only 42 of 263 individuals (16\%) had normal mucosa when gastric biopsies where performed: $50 \%$ had superficial gastritis and $34 \%$ had atrophic gastritis [15]. Atrophic gastritis is a risk factor for gastric cancer, especially when extensive and warrants endoscopic surveillance with multiple biopsies and OLGA/OLGIM staging [16].
Also, in a recent systematic review of population based endoscopic screening on 3063 individuals from Sweden, Italy and China (symptomatic and asymptomatic, ages 18-80), EGD revealed the prevalence of esophagitis in $6.4 \%$ to $15.5 \%$ of cases and Barrett esophagus with confirmed intestinal metaplasia from $1.3 \%$ to $1.6 \%$. The same patients had associated gastroduodenal erosions in $5.3 \%$ to $49.9 \%$ of cases, peptic ulcer in $4.1 \%$ to $17.2 \%$ cases and gastric neoplasia in $0.1 \%$ to $1.1 \%$ of cases [17].

Are these gastro-duodenal findings clinically significant? Except for gastric neoplasia, the importance of other lesions is difficult to quantify. Although, it has been noted that Helicobacter pylori infection seem to protect against Barrett's esophagus development, it is safe to presume that if Barrett's esophagus and gastric neoplasia risk factors are similar (older age, smoking, obesity), both lesions incidence should be increased in a high risk population $[18,19]$.

So, where are we with the findings from this paper?

We need to weigh the advantages of a presumed higher participation in community setting (not confirmed here [1]) and the ease of non-sterilization with the disadvantages of less than $100 \%$ ability to biopsy and not examining the gastroduodenal mucosa. Cost efficacy analysis taking into account all these variables are warranted.

\section{Competing interests}

None

References

[1] Crews NR, Gorospe EC, Johnson ML et al. Comparative quality assessment of esophageal examination with transnasal and sedated endoscopy. Endoscopy International Open 2017; 05: E340 - E344

[2] Sami SS, Dunagan KT, Johnson ML et al. A randomized comparative effectiveness trial of novel endoscopic techniques and approaches for Barrett's esophagus screening in the community. Am J Gastroenterol 2015; 110: $148-158$

[3] Sami SS, Ragunath K, lyer PG. Response to Syed et al. Am J Gastroenterol 2015; 110: $937-938$

[4] ASGE guidelines. The role of endoscopy in Barrett's esophagus and other premalignant conditions of the esophagus. Gastrointestinal Endoscopy: 2012; 76: 1087-1094

[5] Fitzgerald RC, di Pietro M, Ragunath K et al. British Society of Gastroenterology guidelines on the diagnosis and management of Barrett's esophagus. Gut 2014; 63: 7-42

[6] Shaheen NJ, Falk GW, lyer PG et al. ACG Clinical Guideline: Diagnosis and Management of Barrett's Esophagus. Am J Gastroenterol 2016; 111: $30-50$

[7] Lin OS, Schembre DB, Mergener K et al. Blinded comparison of esophageal capsule endoscopy versus conventional endoscopy for a diagnosis of Barrett's esophagus in patients with chronic gastroesophageal reflux. Gastrointest Endosc 2007; 65: 577 - 583

[8] Galmiche JP, Sacher-Huvelin S, Coron E et al. Screening for esophagitis and Barrett's esophagus with wireless esophageal capsule endoscopy: a multicenter prospective trial in patients with reflux symptoms. Am J Gastroenterol 2008; 103: 538-555 
[9] Ramirez FC, Akins R, Shaukat M. Screening of Barrett's esophagus with string-capsule endoscopy: a prospective blinded study of 100 consecutive patients using histology as the criterion standard. Gastrointest Endosc 2008; 68: 25-31

[10] Paterson AL, Lao-Sirieix P, O'Donovan M et al. Range of pathologies diagnosed using a minimally invasive capsule sponge to evaluate patients with reflux symptoms. Histopathology 2017; 70: $203-210$

[11] Jobe BA, Hunter JG, Chang EY et al. Office-based unsedated small-caliber endoscopy is equivalent to conventional sedated endoscopy in screening and surveillance for Barrett's esophagus: a randomized and blinded comparison. Am J Gastroenterol 2006; 101: 2693-2703

[12] Shariff MK, Bird-Lieberman EL, O'Donovan M et al. Randomized crossover study comparing efficacy of transnasal endoscopy with that of standard endoscopy to detect Barrett's esophagus. Gastrointest Endosc 2012; 75: 954-961

[13] ASGE status evaluation report. Equipment for pediatric endoscopy. Gastrointestinal Endoscopy; 2012; 76: 8-17

[14] Sami SS, Subramanian V, Ortiz-Fernández-Sordo J et al. Performance characteristics of unsedated ultrathin video endoscopy in the assessment of the upper GI tract: systematic review and meta-analysis. Gastrointest Endosc 2015; 82: 782 - 792
[15] Roman LD, Lukyanchuk R, Sablin OA et al. Prevalence of H. pylori Infection and Atrophic Gastritis in a Population-based Screening with Serum Biomarker Panel (GastroPanel $\left.{ }^{\circledR}\right)$ in St. Petersburg. Anticancer Res 2016; 36: 4129-4138

[16] Dinis-Ribeiro M, Areia M, de Vries AC et al. Management of precancerous conditions and lesions in the stomach (MAPS): guideline from the European Society of Gastrointestinal Endoscopy (ESGE), European Helicobacter Study Group (EHSG), European Society of Pathology (ESP), and the Sociedade Portuguesa de Endoscopia Digestiva (SPED). Endoscopy 2012; 44: $74-94$

[17] Zagari RM, Eusebi LH, Rabitti S et al. Prevalence of upper gastrointestinal endoscopic findings in the community: A systematic review of studies in unselected samples of subjects. J Gastroenterol Hepatol 2016; 31: $1527-1538$

[18] Rubenstein JH, Inadomi JM, Scheiman J et al. Association between Helicobacter pylori and Barrett's esophagus, erosive esophagitis, and gastroesophageal reflux symptoms. Clin Gastroenterol Hepatol 2014; 12: $239-245$

[19] Tahergorabi Z, Khazaei M, Moodi M et al. From obesity to cancer: a review on proposed mechanisms. Cell Biochem Funct 2016; 34: 533 545 